\title{
Phasage des travaux et organisations transitoires : quels rôles pour l'ergonome?
}

scheduling building work and temporary organisation: how can the ergonomist contribute?

François Daniellou, Jacques Escouteloup et Joffrey Beaujouan

\section{OpenEdition}

Journals

Édition électronique

URL : https://journals.openedition.org/activites/2503

DOI : 10.4000/activites.2503

ISSN : $1765-2723$

Éditeur

ARPACT - Association Recherches et Pratiques sur les ACTivités

Référence électronique

François Daniellou, Jacques Escouteloup et Joffrey Beaujouan, «Phasage des travaux et

organisations transitoires : quels rôles pour l'ergonome? », Activités [En ligne], 8-1 | avril 2011, mis en ligne le 15 avril 2011, consulté le 21 septembre 2021. URL : http://journals.openedition.org/activites/ 2503 ; DOI : https://doi.org/10.4000/activites.2503 


\title{
Phasage des travaux et organisations transitoires : quels rôles pour l'ergonome?
}

\author{
Joffrey Beaujouan \\ Université de Bordeaux, Institut Polytechnique de Bordeaux, ENSC, Département d'Ergonomie, 146 rue Léo-Saignat \\ 33076 Bordeaux CEDEX \\ joffrey.beaujouan@ensc.fr
}

Jacques Escouteloup

Université de Bordeaux, Institut Polytechnique de Bordeaux, ENSC, Département d'Ergonomie jacques.escouteloup@orange.fr

François Daniellou

Université de Bordeaux, Institut Polytechnique de Bordeaux, ENSC, Département d'Ergonomie francois.daniellou@ensc.fr

\begin{abstract}
Scheduling building work and temporary organisation: how can the ergonomist contribute? Extensions to occupied premises are one of the major categories of architectural situation, raising the issue of coordinating construction work with the continuous operation of the establishment. The works schedule is often determined by the architects on the basis of technical and administrative constraints. In many cases, temporary organisation methods to ensure smooth operation during building are not planned in advance. Ergonomics has often focused on understanding the shift from Situation A to Situation B in an architectural context but has devoted less attention to the intermediate stages between A and B and their impact on occupants' health and the efficiency of the establishment. This paper examines various examples of this type of project and suggests how ergonomists may help to reconcile continued operation and building work by: (1) encouraging the two professional groups to meet and discuss the issues, (2) using simulations to determine the broad outlines of the schedule, (3) helping occupants to define temporary organisation methods and (4) adjusting those forms of organisation to major stages in the schedule.
\end{abstract}

KEYWORDS

Reflective practice, ergonomic intervention, architectural design, works scheduling, temporary organisation, professional groups.

\section{Introduction}

Il n'est pas rare qu'un bâtiment construit fasse l'objet au cours de son histoire de transformations successives. Le développement, la modernisation, ou encore la réorganisation d'un établissement sont autant de raisons possibles conduisant à ces modifications. Citons quelques cas rencontrés dans des projets récents comme l'augmentation de la capacité d'accueil d'une maison de retraite et la 
construction d'un accueil de jour, la mutualisation successive de services d'une structure hospitalière, la restructuration de blocs opératoires, la restructuration et l'extension de maternités ou d'unités gériatriques, la modernisation de processus industriels à l'intérieur d'usines de vinification anciennes.

Restructurations et extensions de bâtiments touchent de nombreux domaines et font partie des grandes classes de situations de conception existantes. Ces transformations se réalisent parfois au cœur d'un « existant occupé » pendant la réalisation des travaux, ce qui pose de nombreuses difficultés dans «l'utilisation transitoire du bâtiment » entre l'installation du chantier et son retrait. Cette situation met en évidence une problématique de fond dont nous souhaitons discuter à partir d'expériences de terrain: comment concilier continuité de fonctionnement d'un établissement et réalisation de travaux dans le cadre d'un projet de restructuration ou d'extension architecturale? Quelles sont les répercussions du chantier sur le fonctionnement de la structure? Quelles sont les conséquences sur les utilisateurs et sur la performance de l'établissement pendant la phase «transitoire » de réalisation de l'ouvrage?

Le cadre de référence de nos propos repose sur plusieurs interventions ergonomiques réalisées dans des projets d'extension et de restructuration architecturale dans le domaine sanitaire et social. Cela n'exclut nullement les recouvrements et transferts possibles à d'autres domaines et dans un cadre industriel. La posture choisie dans cet article est celle de la «pratique réflexive » (Argyris \& Schön, 1974) qui vise à capitaliser les actions réalisées - dans notre contexte par les ergonomes - en vue de mettre en tension des modèles existants avec les descriptions et propositions de modélisation ainsi produites. La réflexion dans l'action y est envisagée comme une activité de recherche (par exemple, Argyris, 1983, Saint-Arnaud, 1992) contribuant à l'enrichissement des modélisations de l'activité transformatrice de l'ergonome. Le recueil des faits constatés, mais également l'étude du rôle de l'équipe d'ergonomes dans l'action de transformation ont été possibles à partir de l'analyse croisée de différentes données recueillies au cours de l'intervention (Petit, Querelle, \& Daniellou, 2007). Celles-ci concernaient notamment les comptes rendus de réunions, les documents produits par les ergonomes et par les autres acteurs du projet, des mesures réalisées in situ avec des outillages spécifiques, des «données d'époque » formalisées dans un carnet de bord, etc.

La première partie de l'article s'attache à développer la problématique du chantier sur un site occupé par la mise en perspective de différents constats réalisés « in situ » qui ont la particularité et l'intérêt au regard de la problématique étudiée de combiner à la fois l'analyse de situations de travail et de vie dans l'usage « transitoire » d'un bâtiment en cours de restructuration et d'extension. Nous préciserons dans ces circonstances les notions de «phasage des travaux » et «d'organisations transitoires », puis nous ferons état de la façon dont les projets sont structurés pour articuler continuité de fonctionnement et travaux lorsque cette configuration se présente en détaillant les problèmes que cela pose.

La deuxième partie de l'article se centre sur le développement d'une démarche de travail possible de l'ergonome dans un contexte de travaux sur site occupé. Cette proposition, qui ne vise pas l'exhaustivité, sera illustrée par différents exemples concrets, extraits d'une des interventions ergonomiques récentes conduites dans ces circonstances. L'ergonomie s'est attachée à développer des concepts, des méthodes et des démarches d'intervention pour comprendre, participer à et influencer les processus de conception (par exemple, Béguin, 2007a; Daniellou, 2004; Martin, 2004). Les ergonomes ne se contentent plus d'apporter des informations et des recommandations issues des analyses du travail, ils cherchent également à influencer la manière dont les projets sont conduits et, parfois le processus de conception lui-même. Les suggestions proposées s'inscrivent dans cette orientation.

\section{1.- Phasage et organisations transitoires dans les projets architecturaux}

\section{1.- Phasage des travaux sur site « occupé »}

Ce titre suggère de préciser ce que nous entendons par «phasage des travaux » en spécifiant par la 
suite les raisons de sa réalisation sur un site « occupé ».

Le cadre dans lequel se déroulent les projets architecturaux est très fortement marqué par la loi sur la Maîtrise d'Ouvrage Publique (MOP). La réalisation des travaux correspond à la phase de « chantier » qui précède la réception de l'ouvrage et son démarrage non stabilisé (Armand, Raffestin, \& Couffignal, 2001). La conception d'un ouvrage et son exécution sont sous la responsabilité du Maître d'Euvre, défini comme la personne physique ou morale qui par sa compétence, est chargée par le Maître d'Ouvrage ou la personne responsable du marché de diriger et contrôler l'exécution des travaux (Estingoy \& Rabatel, 1994). Les missions de la Maîtrise d'CEuvre comprennent à la fois une phase d'étude, de contrôle, mais également de coordination et de réception des travaux.

La réalisation des travaux va être ordonnancée, coordonnée et pilotée à partir d'un " phasage technique ». Il vise notamment, du point de vue de l'ingénierie, la définition et l'organisation temporelle et spatiale des interventions techniques des différents corps de métier, ceux qui réalisent la structure porteuse de l'ouvrage et ceux qui équipent le bâtiment (Six, 2004). Ces travaux se matérialisent par l'installation du chantier jusqu'à son retrait (Armand et al., 2001). La mise en place de matériels plus ou moins lourds (grue, bétonnières, etc.) exige l'aménagement des lieux permettant de les recevoir dans de bonnes conditions. L'utilisation de surface de stockage de matériaux et matériels multiples, les besoins en fluides (eau, éclairage en puissance supérieure par exemple), les approvisionnements et les évacuations de matière par véhicule attirent l'attention sur le fait que le chantier a ses propres exigences de fonctionnement. Six (2004) souligne en effet que la complexité d'un chantier à travers l'anticipation des questions liées à l'enchaînement des tâches, au respect du planning, à la logistique, doit être au centre des réflexions qui alimentent le processus de conception. La conception est considérée ici comme un ensemble non homogène comportant une partie relative à l'ouvrage à construire ou à restructurer, et une autre relative au chantier, le précédant et accompagnant sa réalisation. Il s'agit « d'aborder le chantier non plus seulement comme l'étape ultime d'un processus comportant une phase de conception et une phase de réalisation, mais de lui (re)donner une place centrale au sein d'une véritable conduite de projet » (Six, 2004, p. 637).

Plusieurs raisons possibles sont susceptibles d'être à l'origine de travaux à «l'intérieur d'un existant occupé ». Elles peuvent tout d'abord être d'ordre économique (par exemple, rentabiliser les installations, répondre au calendrier de production). Il s'agit alors d'assurer la continuité de production d'un bien ou d'un service pendant le chantier. La faisabilité ou non « d'exporter » l'activité hors des zones de réalisation des travaux peut expliquer également ce choix de combiner continuité de fonctionnement et chantier. À un autre niveau, d'autres facteurs peuvent expliquer la nécessaire continuité des missions dévolues à l'établissement pendant les travaux. Elles sont étroitement liées aux caractéristiques du bien ou du service produit. Par exemple, dans les activités de processus continus, ou encore lorsque l'objet du travail est étroitement lié à une rythmicité d'ordre biologique dont il faut assurer la permanence.

Les expériences sous-jacentes à nos propos s'inscrivent plus particulièrement dans les dernières circonstances évoquées, et à l'intérieur desquelles les exigences du travail impliquent d'assurer différentes fonctions dont certaines sont vitales telles que la continuité des soins à procurer, la distribution des repas ou encore la réalisation des toilettes.

Les travaux sur site occupé vont modifier temporairement les possibilités d'utilisation du bâtiment (Figure 1) et avoir des conséquences plus ou moins favorables sur la permanence du fonctionnement de l'établissement. 
Continuité des prestations à assurer par la structure (échelle de temps)

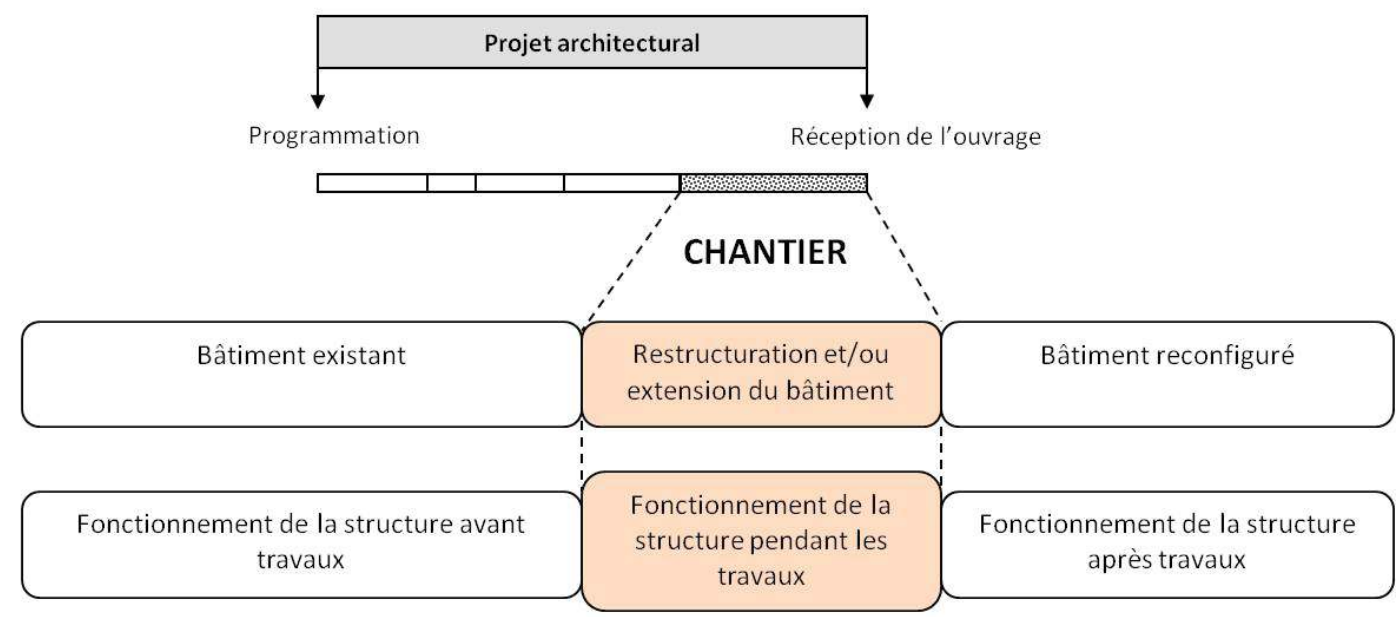

Figure 1: Modification du fonctionnement de la structure pendant la réalisation des travaux

Figure 1: Changes in the operation of the establishment during building work.

La volonté de permanence du fonctionnement pendant les travaux est l'une des composantes possibles d'un projet qui ne porte pas sur le seul bâtiment, mais sur un mode de fonctionnement du système (Martin, Escouteloup, \& Daniellou, 1996; Ledoux, 2000), c'est-à-dire un futur à faire (Ledoux, 2000) et qui concerne notamment le fonctionnement « transitoire » de l'établissement.

Le cas d'une extension-restructuration sur un site inoccupé par exemple à la suite de "l'exportation » des situations de travail et de vie pendant la réalisation des travaux n'a pas été retenu ici puisqu'il s'éloigne de la problématique de fond dont nous souhaitons discuter bien qu'il existe des parentés et zones de recouvrements possibles avec notre objet d'étude. Également, et pour des raisons similaires, nous n'évoquerons qu'indirectement dans cet article la problématique centrale de l'optimisation des conditions de réalisation des travaux par leur anticipation dans le processus de conception de l'ouvrage.

\section{2.- Impacts des travaux sur le fonctionnement de l'établissement}

Deux fonctionnements aux exigences différentes vont devoir cohabiter et s'articuler pendant la réalisation de travaux à l'intérieur d'un site occupé. Celui relatif à la continuité de production de biens ou de services dévolue à l'établissement, et celui lié aux exigences du chantier, à travers le séquencement temporel et spatial des opérations de travaux.

Cette cohabitation va engendrer des co-activités qui vont venir perturber de façon plus ou moins marquée les situations d'utilisation du bâtiment.

\subsection{1.- Classes de problèmes rencontrés dus à la co-activité}

Le caractère invasif des travaux entraîne des contraintes qui, si elles ne sont pas anticipées, vont être révélées au cours du chantier et affecter de façon plus ou moins importante le fonctionnement de l'établissement. Plusieurs classes de problème ont été constatées:

- modification des accès et des circuits;

— surface d'un « espace fonction » diminuée ou supprimée;

- proximités et croisements dans des zones incompatibles; 
- modifications des ambiances physiques, production de nuisances.

Les travaux peuvent avoir des impacts importants sur les circuits verticaux et horizontaux de l'établissement. Un circuit de matière ou de personnes peut être coupé par les exigences de délimitation du chantier. Cela peut modifier les points de livraison possible (repas, linge, médicaments par exemple) ou encore les zones de ramassage des déchets. Les entrées modifiées sont sources de perturbations pour les visiteurs, les livreurs. La modification des circuits s'est traduite au cours d'un projet par l'isolement de certains résidents, empêchant la distribution des repas et bloquant leurs déplacements vers des salles d'activités.

La délimitation du chantier peut provoquer également la diminution ou la suppression provisoire d'un « espace fonction ». Nous étions confrontés sur un projet à une réduction très importante de la surface de la salle de restauration de l'établissement, « amputée » par une zone occupée par le chantier, sans pour autant disposer d'espace supplémentaire ailleurs pour réaliser dans de bonnes conditions la distribution des repas.

Par ailleurs, la co-activité peut être à l'origine de proximités et de croisements dans des zones difficilement compatibles entre opérateurs, usagers et ouvriers. Cela peut se traduire par exemple par une proximité trop importante entre les zones propres soumises à des exigences strictes d'hygiènes (cuisines, blocs opératoires, etc.) et les zones de chantier générant des poussières et particules multiples. Des briques ont pu être retrouvées sur des chariots de soin, ou encore des ouvriers ont été obligés de rentrer à l'intérieur de chambres occupées pour réaliser des travaux. Ces situations rendent compte de la difficulté d'articuler dans l'espace et le temps une multiplicité de situations de travail ou de vie aux exigences parfois contradictoires. Dans de nombreux cas, ces problématiques ont pour origine la délimitation du chantier mal pensée car ne permettant pas aux ouvriers de réaliser correctement leur stockage de matériel de construction (souvent encombrant et difficilement manipulable). Les régulations mises en œuvre par les ouvriers consistent à prendre des accès réservés aux résidents par exemple, ou utiliser des zones de stockage dédiées au fonctionnement de l'établissement.

Le chantier va entraîner également des modifications importantes des ambiances physiques à proximité des travaux. Concernant les ambiances acoustiques, des mesures réalisées montrent qu'à certains moments de la journée le niveau de bruit peut atteindre plus de 100 décibels (mesure en crête) à l'intérieur d'une chambre de patient occupée pendant des travaux, ou encore dans les couloirs d'un bâtiment en chantier. Plusieurs sources sont à l'origine de ces bruits pour certains quasi continus et générés dans des plages horaires décalées: les machines utilisées pour le chantier (marteaupiqueur, meuleuses, scies), les chutes de matière et leur évacuation (démolition de murs), ou encore les approvisionnements de matériaux et matériels. L'impossibilité d'entendre les appels malades pour les soignants, ou encore les difficultés de retrouver des périodes de calme et de repos pour des résidents avancés en âge témoigne du caractère « dégradé » de la période de travaux. Les conditions de réalisation du travail des professionnels et les conditions de vie des résidents en sont totalement bouleversées. Le chantier va augmenter considérablement la production de poussières, avec des pénétrations possibles dans les espaces de travail et de vie. Poussières, bruits et odeurs vont contribuer à la fermeture des fenêtres par les utilisateurs produisant des effets indirects sur les ambiances thermiques. Les ambiances lumineuses peuvent également être modifiées considérablement, par exemple par l'obstruction des sources lumineuses naturelles proches des zones de travaux pour prévenir des projections de matière.

Les contraintes évoquées vont venir modifier les conditions d'utilisation du bâtiment pendant la période des travaux de façon superficielle ou beaucoup plus profonde entraînant des conséquences plus ou moins positives sur les hommes et la performance de l'établissement.

\subsection{2.- Conséquences sur les hommes et la performance de l'établissement}

Trois catégories d'utilisateurs potentiels du bâtiment pendant la réalisation des travaux peuvent être distinguées. Ceux qui y travaillent au quotidien pour assurer les missions dévolues à l'établissement, 
les opérateurs ; ceux qui l'utilisent dans un cadre non professionnel et jouissent des services proposés, les usagers (par exemple Petit, 2005); et enfin ceux qui y travaillent pour réaliser des travaux, que nous appellerons les professionnels de la construction (Six, 2004). L'adéquation entre les exigences de continuité de fonctionnement de l'établissement et les caractéristiques des travaux à partir du phasage proposé est essentielle puisqu'un couplage difficile de ces deux composantes est à l'origine de nombreuses difficultés schématisées dans la figure 2. Les utilisateurs sont contraints de trouver des formes d'adaptations coûteuses, car immédiates et bien souvent improvisées. Des effets négatifs y sont associés sur leur propre santé, sur la performance de l'établissement et celle du chantier.

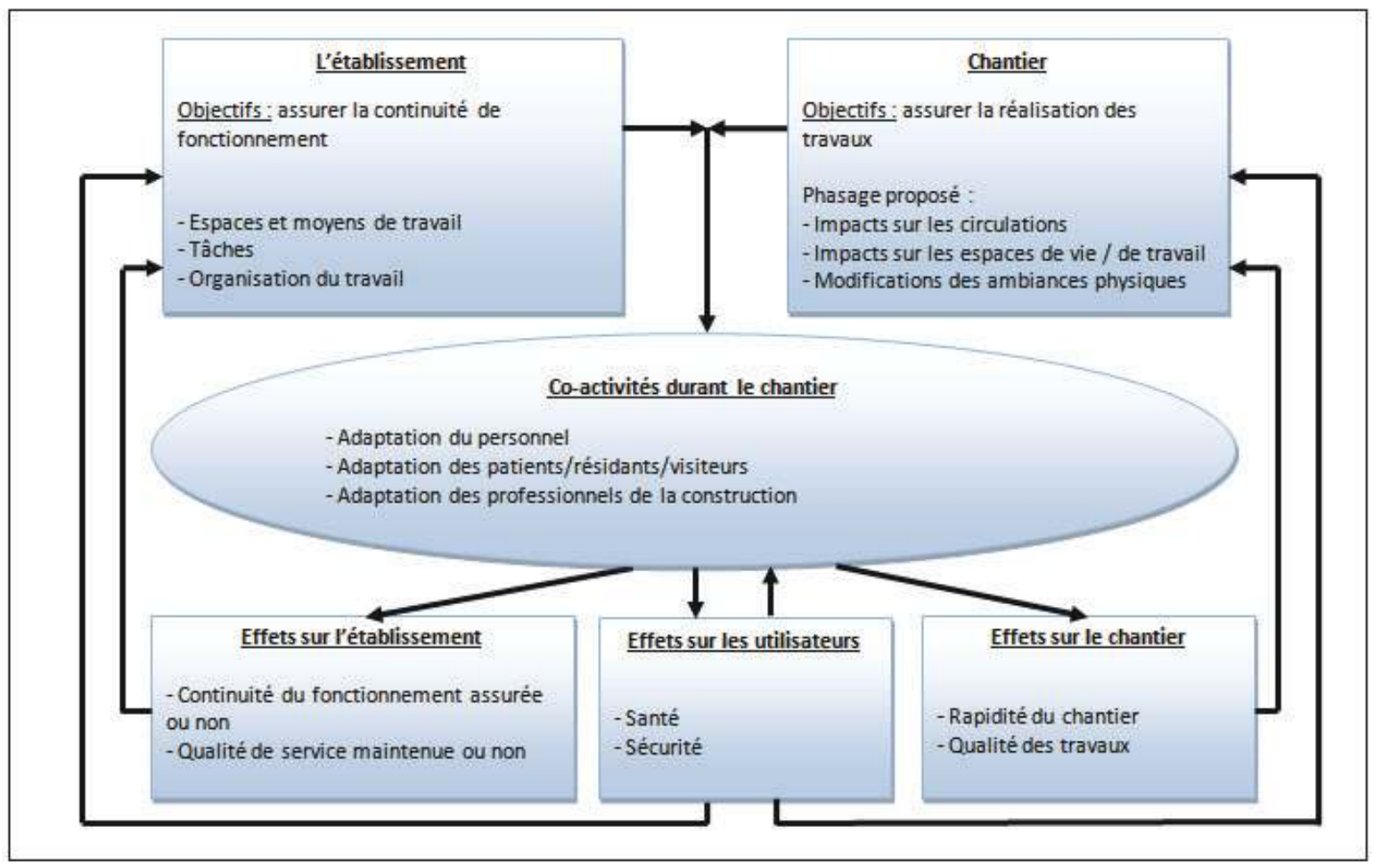

Figure 2: Couplage entre phasage des travaux et fonctionnement de l'établissement

Figure 2: Coordinating the building schedule with the operation of the establishment.

Petit et Escouteloup (2009) ont identifié des conséquences de plusieurs ordres sur les hommes constatés à la suite d'un couplage difficile du chantier avec la permanence de fonctionnement de l'établissement:

- des effets physiologiques liés au bruit, à la poussière produisant l'augmentation avérée de gênes respiratoires, oculaires, des maux de tête et des troubles du sommeil des opérateurs et usagers ;

- des effets psychiques liés au sentiment de faire un travail de mauvaise qualité au regard des conditions de réalisation de la tâche dégradées par le chantier;

- des effets sociaux sur les collectifs d'opérateurs liés à la désorganisation non anticipée (par exemple des altercations entre collègues pour effectuer un rapprochement entre une mère avec son enfant séparé par le chantier se sont produites au cours de l'extension d'une maternité);

— des effets sociaux sur les usagers mettant à mal l'équilibre et la rythmicité de leur vie collective.

L'imbrication entre permanence de fonctionnement et chantier est plus ou moins réussie dans les projets d'extension et/ou restructuration architecturale. La notion d'organisations transitoires nous semble dans ces circonstances essentielle pour limiter les effets négatifs constatés sur l'établissement, le chantier et les utilisateurs pendant la période de travaux. 


\section{3.- Qu'entend-on par « organisations transitoires » ?}

Au cours du chantier, les possibilités d'utilisation du bâtiment sont évolutives. Les orientations de phasage ferment, mais ouvrent également des pans entiers de possibilité aux situations d'utilisations futures du bâtiment pendant la réalisation des travaux. La progression de l'ouvrage par zone et par période va constituer tantôt une contrainte et tantôt une ressource dans ses formes d'utilisation transitoire. La création de nouveaux circuits « potentiellement» utilisables pendant que d'autres sont coupés, ou encore la construction de nouvelles surfaces disponibles alors que d'autres sont amputées, sont autant d'exemples rencontrés au cours d'un chantier. La notion d'organisation transitoire peut être précisée selon plusieurs perspectives complémentaires pour délimiter ses frontières.

Du point de vue de sa finalité, elle vise la définition d'orientations « provisoires » de fonctionnement pour assurer sa continuité pendant les différentes opérations de travaux. La notion d'organisation transitoire ne se limite pas aux seuls leviers organisationnels (moyens humains, nouvelles procédures). Elle concerne également la modification des moyens techniques à disposition des utilisateurs.

Les acteurs susceptibles d'en décider les modalités sont principalement ceux qui sont garants de la continuité du fonctionnement de l'établissement pendant le chantier (dans notre contexte d'intervention il s'agit notamment des directeurs, des médecins et des cadres de santé). En ce sens, les organisations transitoires sont généralement portées par les acteurs de la Maîtrise d'Ouvrage.

Du point de vue de sa temporalité, une organisation transitoire est limitée. Elle s'inscrit entre un état initial (organisation avant travaux) et un état final (organisation projetée après travaux) en passant selon les cas par un nombre plus ou moins important d'états intermédiaires successifs.

Du point de vue de sa dynamique, elle est intrinsèquement liée au caractère évolutif de l'avancement des travaux réalisés par phase. Ceci explique qu'il existerait non pas une, mais autant d'organisations transitoires à définir que de modifications des propriétés d'un bâtiment au cours des opérations de chantier remettant en cause l'organisation précédente. On ne fabrique plus les repas au même endroit car la cuisine fait l'objet d'une modernisation, on ne lave plus le linge dans la même zone car la lingerie est déplacée, ou encore on ne peut plus distribuer les repas par le circuit habituel, car il sera coupé pendant l'une des phases des travaux.

\section{4.- Phasage des travaux et organisations transitoires dans les projets}

Plusieurs interventions ergonomiques réalisées dans un contexte de restructuration et/ou d'extension sur site occupé invitent à formuler différents constats sur la prise en compte du phasage des travaux et des organisations transitoires dans les processus de conception. Ceux-ci dépendent du moment où le phasage est défini dans la conduite du projet, des caractéristiques des phasages proposés, et enfin des modalités d'information et de transmission du phasage par les concepteurs aux utilisateurs (en dehors des professionnels de la construction).

Deux configurations « extrêmes » ont été rencontrées en ce qui concerne le moment où le phasage est envisagé dans la conduite du projet.

1/ Dans la première configuration il est défini après les plans détaillés du futur ouvrage, c'est-à-dire très tardivement dans la conduite de projet. Le phasage est considéré comme secondaire et non prioritaire dans les premières étapes du processus de conception. Ce qui l'est concerne essentiellement les modalités de restructuration ou d'extension du bâtiment (faisabilité, respect du programme architectural notamment). La réalisation des travaux est dans cette configuration une variable d'ajustement fixée à partir de plans détaillés ayant cristallisé (Béguin, 2007b) les différentes orientations de conception. Six (2004) souligne d'ailleurs que de nombreuses difficultés rencontrées au cours du chantier ont pour origine cette séparation très marquée entre la phase de conception de l'ouvrage et les exigences concernant sa réalisation concrète pendant les travaux.

2/ Dans la seconde configuration, le phasage fait l'objet d'une prise de conscience importante des 
acteurs du projet, bien souvent pour des raisons liées à la complexité et la faisabilité de l'opération. Il est envisagé de façon précoce dans la conduite de projet, parfois dès l'étape du concours architectural où une proposition de phasage à partir du plan d'esquisse proposé est exigée par le Maître d'Ouvrage. Le phasage des travaux n'est pas une variable d'ajustement des choix de conception cristallisés dans le plan, il est davantage considéré comme l'une des composantes essentielles du projet et va venir influencer les orientations de conception.

Ainsi, il existe un continuum entre ces deux pôles concernant le moment de prise en compte du phasage des travaux dans la conduite du projet. D’un côté il est envisagé tardivement, étant considéré comme une simple variable d'ajustement aux orientations de conception stabilisées. De l'autre, il est considéré de façon beaucoup plus précoce et influence de façon importante la conception, pour des raisons liées à la faisabilité technique de l'opération ou encore dans un souci d'optimisation du coût économique de réalisation de l'ouvrage.

Le deuxième constat concerne les caractéristiques des phasages proposés par les équipes de Maitrise d'Euvre, principalement guidées à partir de leur composante technique, réglementaire et économique. L'aspect technique concerne principalement la faisabilité de réalisation effective des travaux (accessibilité, possibilités d'approvisionnement et d'évacuation des matières par exemple), la coordination temporelle et spatiale des différents corps de métier de l'installation du chantier jusqu'à sa clôture (Armand et al., 2001). La composante réglementaire concerne l'ensemble des législations applicables au chantier, par exemple en matière de santé et sécurité. La composante économique est présente transversalement, elle vise l'optimisation des coûts relatifs aux opérations de travaux (durée des travaux, nature des travaux à réaliser, moyens techniques et humains nécessaires). La définition du phasage à partir des seules composantes évoquées met en évidence un écueil souligné par Daniellou (2004) dans la conduite de projet, celui de son pilotage par sa composante technique réglementaire et économique plutôt que par ses aspects fonctionnels et organisationnels. Les conséquences du phasage sur ces derniers aspects sont souvent méconnues et sous-estimées par les concepteurs.

Le dernier constat concerne les modalités d'information et de transmission du phasage par les concepteurs aux utilisateurs (personnels, patients, familles, etc.) et qui constitue, de notre point de vue et pour les projets auxquels nous avons participé, un obstacle important à la construction « d'un monde commun » (Béguin, 2007a) visant à rendre compatible la permanence du fonctionnement de l'établissement et la réalisation des travaux pendant le chantier dans les meilleures conditions. Nous constatons l'absence ou la très faible participation des utilisateurs dans l'instruction des orientations de phasage retenues, notamment ceux ayant la responsabilité du fonctionnement de l'établissement ou qui sont chargés d'assurer son opérationnalité pendant les travaux. Par ailleurs, la difficulté de compréhension et d'exploitation par le personnel des outils de représentation du phasage élaborés par les concepteurs, lorsqu'il est transmis tel quel, constitue une autre barrière à l'articulation des différentes logiques en présence. Les intentions de phasage sont préfigurées par des supports matériels qui ont des propriétés complexes (Maline, 1994) difficilement exploitables par les utilisateurs. Cet écueil renvoie au défaut d'outillage (conceptuel, méthodologique, etc.) des utilisateurs qui ne sont pas obligatoirement concepteurs de métier (Darses \& Reuzeau, 2004). Plusieurs outils de représentation du futur peuvent être convoqués (plans, maquettages numériques sur CAO, diagrammes de Gantt) et nécessitent pour les acteurs chargés d'assurer la permanence du fonctionnement de l'établissement pendant les travaux de disposer des connaissances nécessaires à leur compréhension. Ces obstacles sont autant de freins à la construction d'un monde commun qui nécessite la convergence entre les acteurs, l'obligation même de confronter sur une même scène leurs logiques et leurs positions qu'elles soient homogènes ou non (Béguin, 2007a) en vue de concilier continuité de fonctionnement et travaux.

La définition tardive du phasage au cours de la conduite de projet, ses caractéristiques (essentiellement techniques, économiques et réglementaires), ses modalités de transmission aux acteurs chargés d'assurer la permanence du fonctionnement (peu associés, informés à partir de supports difficilement 
compréhensibles) ne facilitent pas l'appréciation de ses impacts sur le fonctionnement de l'établissement. Ces obstacles sont défavorables à l'anticipation des organisations transitoires qui devront bien souvent se définir « sur le tas » (et sur le tard!) pendant les travaux. Dans ces circonstances, les contraintes du chantier « s'imposent » à celles du fonctionnement de l'établissement ayant des effets négatifs sur les utilisateurs et la performance de l'établissement comme nous l'avons souligné à plusieurs reprises.

L'ergonomie s'est beaucoup intéressée à la compréhension de l'évolution d'une situation A vers une situation B transformée dans un contexte architectural. Elle s'est moins préoccupée de la compréhension des états transitoires entre ces deux pôles et ses conséquences sur le plan de la santé des utilisateurs, sur la performance de l'établissement et sur la réussite du projet (Sousa Castro, 2010) notamment lorsque le contexte impose d'assurer la permanence des missions dévolues à la structure pendant le chantier. La deuxième partie de l'article se centrera spécifiquement sur les contributions possibles des ergonomes pour aider à concilier (au mieux) continuité de fonctionnement et travaux dans un contexte d'extension et/ou de restructuration architecturale au cœur d'un site occupé.

\section{2.- Contribution possible des ergonomes}

Favoriser la confrontation entre le monde des concepteurs et celui des utilisateurs, contribuer à instruire les grandes orientations de phasage et accompagner la définition des organisations transitoires par le monde des utilisateurs jusqu'à la fin du chantier sont autant d'opportunités d'actions possibles de l'ergonome pour influencer le contenu et la conduite du projet. Cette partie de l'article propose quatre points de démarche possible de l'ergonome dans un contexte de travaux sur site occupé. Ces propositions issues de nombreuses interventions ergonomiques seront illustrées par différents matériaux empiriques extraits de l'une d'entre elles. Il s'agit d'une intervention réalisée dans le cadre d'un projet d'extension et de restructuration d'une unité gériatrique (UG) au sein d'un hôpital. Un comité de pilotage a été défini (directeurs, médecins, cadres de santé supérieurs notamment) pour suivre l'ensemble des étapes du projet. Le projet pose l'exigence d'une continuité du fonctionnement de la structure pendant la réalisation des travaux. L'équipe d'ergonomes est en situation d'assistance à la Maîtrise d'Ouvrage (AMO) de ce comité de pilotage, dès la définition du projet jusqu'à la prise en main par les utilisateurs futurs des modernisations réalisées. Les missions remplies par les ergonomes tout au long du projet recouvrent un périmètre bien plus large que celui de la problématique évoquée dans cet article.

\section{1.- La rencontre des mondes professionnels}

Concilier la permanence des missions dévolues à l'établissement pendant la réalisation des travaux implique de créer les conditions d'une mise en cohérence des orientations de phasage proposées avec la façon dont l'établissement fonctionne et va pouvoir assurer cette continuité pendant le chantier. Cette articulation est trop complexe pour qu'une seule personne dispose des compétences nécessaires à la résolution des problèmes qui se posent. Le modèle « construction progressive et collective » du processus de conception architectural (Martin, 1998) est transposable à nos propos. Phasage des travaux et organisations transitoires relèvent d'une construction progressive et collective entre un énoncé initial, en particulier la volonté relative au futur qui ne porte pas sur le seul bâtiment, mais sur un mode de fonctionnement (Ledoux, 2000) et sa réalisation. Cette description du déroulement des projets architecturaux replacée dans un contexte de travaux sur site occupé nécessite dans le processus de définition du phasage la mise en place d'une organisation particulière favorable à la confrontation et la coordination de plusieurs mondes professionnels porteurs d'une diversité de sphères de compétence, de logiques et d'identités à préserver. Parmi ces logiques s'expriment d'un côté, la faisabilité technique, économique, réglementaire du phasage, de l'autre, la faisabilité technique et organisationnelle de permanence du fonctionnement de l'établissement. 
Une première mission possible pour l'ergonome vise à favoriser cette rencontre. L'une des conditions préalables à la construction d'une « œuvre commune » est la connaissance partagée des contraintes mutuelles en présence. La transmission des exigences fines du fonctionnement de l'établissement par le Maître d'Ouvrage à l'ensemble des acteurs chargés de la conception et/ou la réalisation à différents niveaux du nouvel ouvrage est essentielle (Six \& Fourot, 2000). Il en est de même en ce qui concerne la transmission précoce, par les acteurs chargés de la conception et/ou la réalisation de l'ouvrage, des connaissances du phasage technique vers les acteurs chargés d'assurer la continuité de fonctionnement de l'établissement.

Sur le projet de l'unité gériatrique, l'équipe d'ergonomes a favorisé le fait que le comité de pilotage et en particulier le responsable du fonctionnement de l'établissement soient demandeurs d'informations précoces auprès de la Maîtrise d'Euvre sur le phasage des travaux. À l'étape du Concours architectural, c'est-à-dire en amont de la sélection du lauréat, il est exigé par le Maître d'Ouvrage la présentation des grandes orientations de phasage retenues par les concepteurs. Sa pertinence est clairement posée comme un critère de sélection. Les propositions de phasage exigées par le comité de pilotage «Maîtrise d'Ouvrage » dès le stade du Concours architectural ont favorisé la prise de conscience de la problématique du phasage au sein de l'établissement occupé. Les ergonomes ont assuré en amont de cette étape du projet une mission essentielle de recensement et de transmission des exigences des principales situations d'action caractéristiques du fonctionnement de l'établissement (Daniellou, 2004) relayées par le comité de pilotage aux équipes de Maîtrise d'Euvre. Cette démarche a contribué à faire en sorte que les exigences liées aux situations de travail et de vie que les concepteurs n'ont aucune raison de connaître, comme le souligne Martin (2004), orientent et soient une source de leur activité (Béguin, 2007b). La figure 3 présente la réponse au Concours de l'une des quatre équipes de Maîtrise d'Euvre concurrentes. La partie consacrée à la réflexion sur le phasage des travaux (zone agrandie de la figure 3) constituait une exigence forte du Concours pesant véritablement sur le choix final du lauréat par le Maître d'Ouvrage.

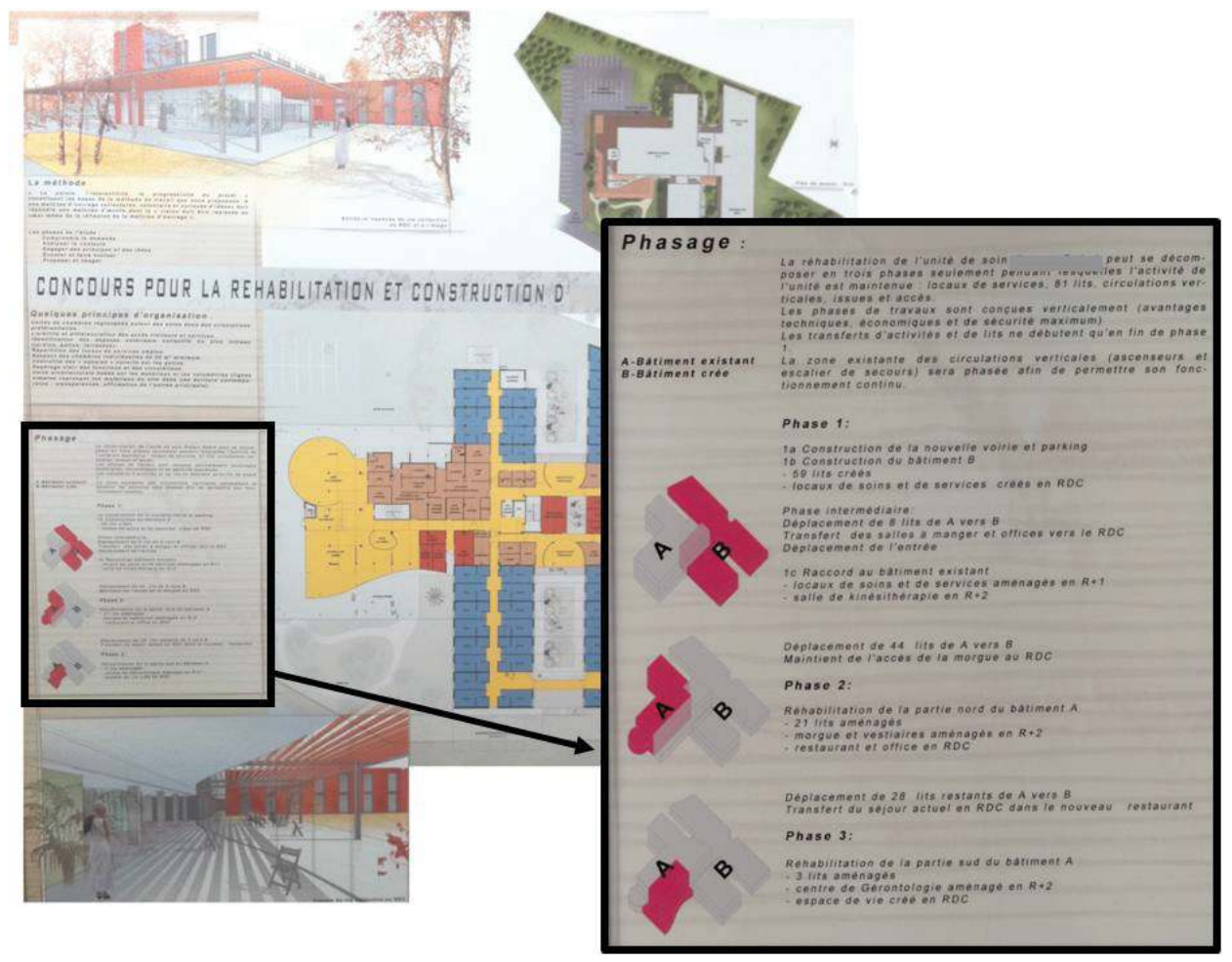

Figure 3: Exemple d'une réponse architecturale de l'un des quatre concurrents.

Figure 3: Architectural solution proposed by one of the four competitors in the contest.

Proposition de phasage et recensement des situations d'action caractéristiques constituent dans 
ce contexte des objets intermédiaires qui participent à la construction de compromis et de savoirs partagés entre les acteurs comme éléments moteurs d'une confrontation sociocognitive (Garrigou, Daniellou, Carballeda, \& Ruaud, 1995) des mondes professionnels et de leur coordination dans un monde commun. Ces objets permettent de déplacer les points de vue des différents individus en présence, et jouent le rôle de référentiel commun définissant un espace pour l'action (Vinck, 2009) délimité par les contraintes du fonctionnement d'un côté et par les contraintes du phasage de l'autre. Dans ces circonstances, le phasage ne serait plus guidé par ses seuls aspects techniques, réglementaires ou économiques, mais deviendrait le résultat de compromis à partir de ses conséquences sur l'établissement du point de vue fonctionnel et organisationnel.

Cette démarche traduit la volonté que, parmi les déterminants de l'élaboration du phasage, puissent apparaître les situations d'utilisation du bâtiment. Il est du ressort de l'ergonome à la fois d'analyser ces situations et également de faciliter leur prise en compte par l'action sur la conduite de projet (Béguin \& Cerf, 2004). L'instruction et la validation des grandes orientations de phasage constituent une deuxième étape de démarche possible.

\section{2.- L'instruction et la validation des grandes orientations de phasage}

L'instruction et la validation du phasage visent à vérifier que les grandes options choisies par les concepteurs ne mettent pas à mal les fonctions et circuits «vitaux » pour la continuité du fonctionnement de l'établissement (l'approvisionnement en médicaments, nourritures et autres matières, la dispense des soins quotidiens, la distribution des repas, ou encore les toilettes pour l'exemple d'une maison de retraite). Il s'agit alors d'anticiper et de prévenir les incidents majeurs (Petit \& Escouteloup, 2009). Cette vérification conduit à explorer les propriétés de l'artefact «phasage » pour prévenir les difficultés associées à sa cristallisation (Béguin, 2007b).

Sur le projet de l'unité gériatrique, les notes d'intention de phasage des quatre concurrents ont fait l'objet d'une analyse détaillée des points sensibles par l'équipe d'ergonomes pour en rendre compte au jury du Concours. Cela a nécessité l'articulation entre l'analyse prospective des situations d'utilisation du bâtiment et de ses exigences pour assurer son fonctionnement, avec une analyse projective de l'intention de phasage. La simulation du phasage et du fonctionnement de l'établissement à partir d'une bibliothèque de situations de vie et de travail élaborée par les ergonomes a permis l'évaluation des intentions de phasage proposées par les concepteurs. La pédagogie des présentations, le nombre de déménagements de résidents pendant les travaux (le plus faible possible), l'impact des nuisances sur les situations de vie et de travail, ainsi que la durée du chantier constituaient plusieurs critères d'instruction des phasages proposés.

Cette démarche a permis la détection de choix cristallisés dans l'artefact des concepteurs qui rendent impossible le fonctionnement transitoire.

Le tableau 1 présente l'analyse d'une note d'intention de phasage restituée au Maître d'Ouvrage pour l'un des quatre candidats au concours architectural.

L'éclairage apporté sur les différentes intentions de phasage a permis d'instruire les avantages et inconvénients des différentes propositions, ouvrant la voie à d'éventuelles modifications du phasage après le concours avec le lauréat.

Cette évaluation peut être réalisée conjointement avec les acteurs garants du fonctionnement de l'établissement. Néanmoins, cela nécessite qu'ils puissent se représenter clairement les étapes de réalisation des travaux. Cette compréhension est étroitement liée aux dispositifs de représentation et de communication choisis, certains étant plus facilement exploitables que d'autres par les utilisateurs (Jeantet, Tiger, \& Vinck, 1996). Cette difficulté rencontrée également dans la définition des organisations transitoires par les utilisateurs est un obstacle de taille à la coordination des mondes professionnels et qu'il s'agit de dépasser. 


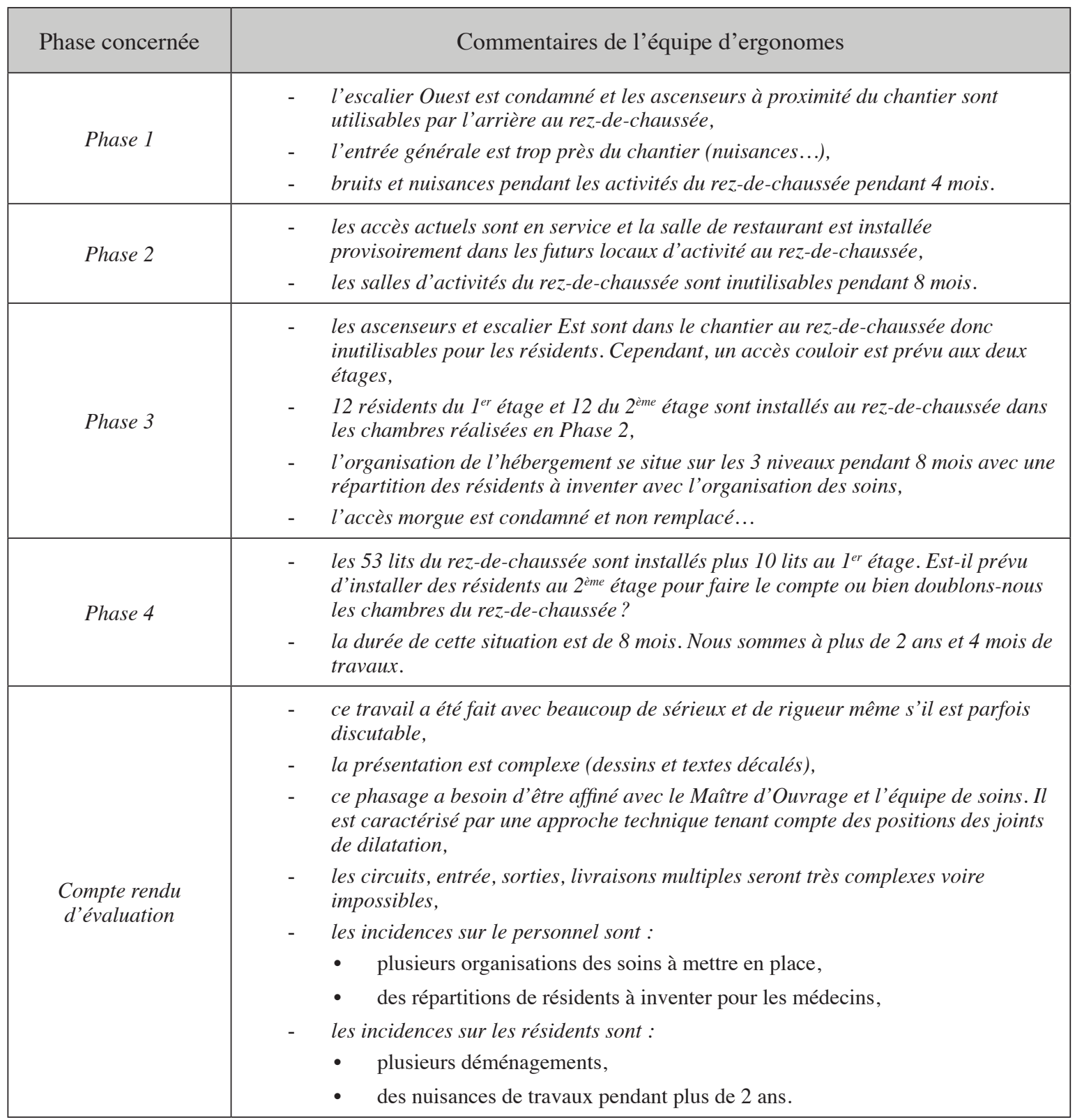

Tableau 1: Analyse des notes d'intention de phasage « équipe B »

Table 1 : Analysis of the proposed scheduling (team B)

\section{3.- La définition des formes possibles d'organisations transitoires}

Concilier continuité de fonctionnement et travaux exige d'articuler deux projets différents. Le projet architectural, duquel découle le phasage des travaux, et le projet d'organisation relatif à l'expression de la volonté de permanence du fonctionnement. Ces projets sont interdépendants puisque l'anticipation des formes possibles d'organisations transitoires va être guidée par les grandes orientations du phasage retenu qui, matérialisées sous forme d'objets intermédiaires, vont prendre le statut de repères pour la conception sur lesquels va s'appuyer l'action (Vinck, 2009). Du côté des concepteurs du bâtiment, l'objet intermédiaire vise l'opérationnalisation des travaux alors que du côté des ingénieurs en organisation il constitue un repère permettant de réinterroger les moyens techniques et humains nécessaires à la permanence du fonctionnement par phase de travaux. La compréhension 
du phasage est un pré requis à la définition des organisations transitoires à la fois pour les hauts responsables de l'établissement, mais également pour ceux qui sont chargés de définir et rendre opérationnel les règles de fonctionnement. La connaissance du phasage pour ces mêmes acteurs facilite la préparation et l'anticipation des modifications transitoires de la sécurité intrinsèque du bâtiment (sécurité incendie, etc.) pour chacune des phases de construction / réhabilitation de l'ouvrage.

Sur le projet de l'unité gériatrique, les ergonomes ont réalisé un travail « d'équipement » (Vinck, 2009) de l'objet intermédiaire «phasage » en vue de favoriser sa compréhension et sa circulation des concepteurs aux membres du comité de pilotage « Maîtrise d'Ouvrage » (direction, médecins, cadres de santé). Ce travail s'est matérialisé par l'introduction de métadonnées facilitant la compréhension de l'objet intermédiaire. Les orientations de phasage transmis par la Maîtrise d'Euvre (plans, diagramme de Gantt) ont été transformées par les ergonomes (Figure 4).

Les étapes des travaux ont été représentées par des figures volumétriques et dynamiques. Les zones concernées par le chantier (en gris) et celles préservées pour assurer la continuité du fonctionnement (en rouge) ont été matérialisées pour chaque phase de travaux prévue. Les circuits préservés et ceux rompus par le chantier ont été mentionnés. Le déplacement d' « espace fonction » tel que l'accueil de jour, ou encore le déménagement de matériels ou de résidents pour libérer des espaces a été spécifié.

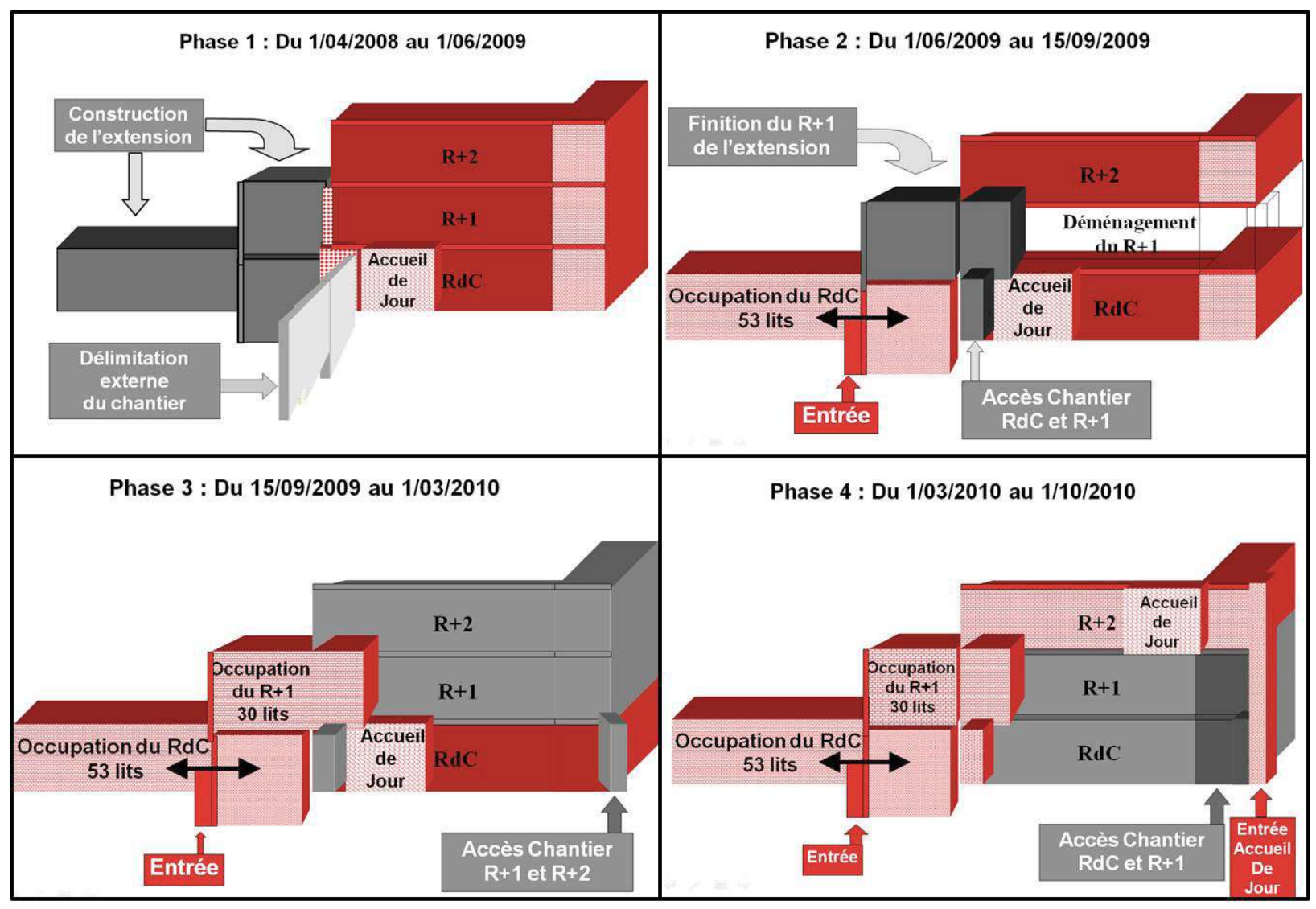

Figure 4: Phasage des travaux reconstruit par les ergonomes à destination des utilisateurs

Figure 4: Works schedule revised by ergonomists for the benefit of users.

L'objet intermédiaire présenté s'est avéré être un support privilégié pour l'anticipation des organisations transitoires. Il a facilité le repérage des chambres en lien direct avec les nuisances, les circuits accessibles et rompus par phase, les surfaces créées et celles qui étaient amputées. À partir de cette représentation, la continuité des situations de travail et de vie a pu être vérifiée; les opérations tiroirs, 
les déplacements des résidents à prévoir ainsi que les organisations des soins par phase ont pu être anticipés. Il en est de même pour les temps incompressibles de déménagement et de réaménagement de l'ensemble du mobilier ou des résidents à transférer. La reconstruction du phasage a également été utilisée pour informer et préparer les familles à s'approprier les configurations transitoires d'utilisation du bâtiment par phase des travaux.

Néanmoins, la représentation volumétrique et par phase de réalisation des travaux a nécessité de façon complémentaire pour certains cadres de santé supérieurs leur présence sur le site existant afin qu'ils visualisent et s'imprègnent bien des limites de chantier, de la réduction des locaux, du passage des camions, de l'exposition des chambres les plus proches du chantier futur. Ceci nous laisserait penser que le seul outil de représentation du phasage reconstruit par l'ergonome, malgré tout ces apports, pourrait être insuffisant pour que la représentation du futur s'élabore de manière pertinente.

Comme le souligne Vicente (1999) anticiper n'est pas prédire. La prédiction des sources de variabilité future, ou encore la prédiction de l'activité d'un sujet susceptible d'inventivité et de créativité posent les limites ontologiques et épistémologiques d'anticiper totalement et pleinement l'activité future (Béguin \& Cerf, 2004). Une anticipation trop forte peut être problématique face aux contingences locales ainsi qu'aux formes de diversité importante des situations d'utilisations du bâtiment et des utilisateurs eux-mêmes pendant la réalisation des travaux. L'anticipation des formes d'organisations transitoires rejoint l'enjeu de l'approche de l'activité future (Daniellou, 2004) et ne vise pas à prévoir en détail l'activité qui s'y déroulera à l'avenir, mais aspire à tendre vers ce que Béguin (2007b) appelle la «plasticité » d'un système sociotechnique et qui consiste à prévoir « l'espace des formes possibles d'activité future » (Daniellou, 1992).

L'action de l'ergonome vise à faciliter la définition d'organisations transitoires plastiques dans lesquelles des formes d'ajustement liées aux aléas soient possibles et à favoriser la mise en place de dispositifs apprenants dans lesquels les techniques de simulation tiennent un rôle essentiel (Béguin \& Pastré, 2002; Pastré, 1999). L'accompagnement de la démarche de simulation des organisations transitoires par phase de travaux conduit dans un même mouvement à vérifier la cohérence des orientations de phasage tout en préparant les utilisateurs à gérer les futures contraintes du chantier.

La non-anticipation des organisations transitoires empêche la possibilité de redéfinir en amont des travaux le phasage dans le cas d'organisations difficilement réalisables et très pénalisantes pour le fonctionnement. Cependant, la fréquence des aléas du chantier constitue un obstacle important puisqu'une modification de dernière minute peut remettre en cause une anticipation de plusieurs mois. Par ailleurs, chaque organisation transitoire vécue par les utilisateurs pendant le chantier constitue sous certaines conditions une opportunité d'apprendre sur ses forces et ses faiblesses en vue d'optimiser les organisations futures. La situation de travail est une occasion d'apprentissage où il s'agit de tirer des enseignements de son expérience dans et par l'organisation (Hatchuel, 1997). Les organisations transitoires anticipées sont ainsi susceptibles de faire l'objet d'ajustements plus ou moins importants au cours du chantier. Nous proposons de développer par la suite quelques éléments de démarche s'étant révélés utiles pour favoriser l'ajustement mutuel des organisations transitoires et des conditions de réalisation des travaux, entre le début et la fin du chantier.

\section{4.- L'ajustement des organisations transitoires pendant le chantier}

Comme le souligne Six (2004), le chantier n'est pas une pure exécution de ce qui est contenu dans des plans. C'est un lieu et un moment où se déploie une importante activité de conception et de régulation de la part des acteurs de l'encadrement et des équipes de compagnons. Les aléas et les difficultés rencontrées au cours des travaux obligent les concepteurs et les entrepreneurs à des modifications dans l'urgence (Martin, 2004), décidées en temps réel et qui peuvent avoir des conséquences importantes sur le fonctionnement provisoire. La personne qui détient la mission d'OPC (Ordonnancement et Pilotage du Chantier) a un rôle essentiel qui consiste notamment à faire circuler l'information, détecter les décisions à prendre, fournir les éléments nécessaires à ces prises de décisions, ou encore 
signaler les éléments qui risquent de perturber le bon déroulement du chantier (Armand et al., 2001). Il est investi de l'autorité du Maître d'EEuvre pour tout ce qui touche le pilotage des travaux.

Sur le projet de l'unité gériatrique, l'équipe d'ergonomes a favorisé la mise en œuvre de boucles de régulations entre les acteurs ayant une connaissance et un niveau de décision suffisant du chantier et des organisations transitoires. La personne ayant la mission d'OPC d'un côté comme interlocuteur principal et de l'autre les acteurs garants du fonctionnement (par délégation cadres de santé, médecins principalement) étaient en relations étroites, alors que bien souvent l'OPC est en lien direct avec les services techniques de l'établissement, rarement avec ceux qui sont porteurs de la logique de continuité de fonctionnement.

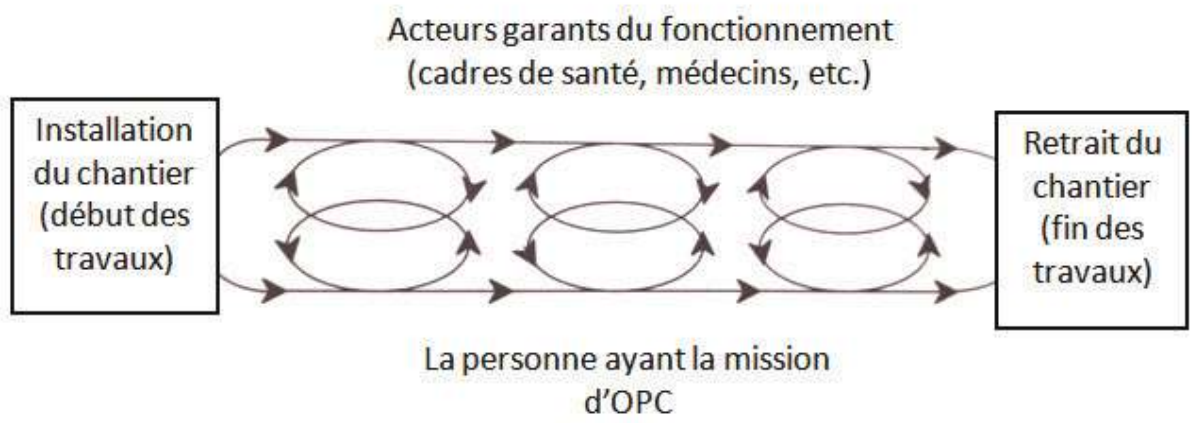

Figure 5: Boucle de régulation au cours du chantier

Figure 5: Feedback loop used during building work.

Ces boucles de régulation ont permis sur le projet d'articuler un nombre important de micros décisions nécessitant un maillage entre celui qui porte l'organisation du chantier et ceux qui sont chargés de l'organisation du fonctionnement de l'établissement. Par exemple, l'installation des circuits des groupes de rafraîchissement nécessitant des interventions lourdes dans des chambres de résidents s'est réalisée pendant les temps d'activité durant lesquels les chambres étaient inoccupées. La présence permanente du cadre de santé dans les réunions de chantier a permis notamment ces coordinations de dernière minute.

Par ailleurs, ces articulations ont facilité également, pour les acteurs chargés du fonctionnement de l'établissement, la transmission, aux acteurs garants de l'organisation du chantier, de l'évolution de certaines contraintes à prévoir suite aux difficultés rencontrées par la mise en place d'organisations transitoires s'étant révélées effectivement trop coûteuses pour le personnel et les patients. Cette possibilité pour l'organisation d'apprendre de son expérience pour devenir plus performante dans l'avenir, c'est-à-dire sa capacité à être « apprenante » (Argyris \& Schön, 1978, 1996) implique notamment la remise en cause constante de l'expérience, sa transformation et sa capitalisation en savoirs accessibles, ainsi que sa diffusion à l'ensemble de l'organisation (Senge, 2000). Ces apprentissages vont avoir des conséquences sur les organisations transitoires futures, susceptibles d'évoluer au cours du chantier. La garantie d'une articulation optimale du fonctionnement de l'établissement avec le déroulement du chantier nécessite dans ce contexte une confrontation des logiques hétérogènes qui puisse perdurer au cours des travaux. Ceci peut être favorisé par le fait que chaque décision prise par les garants de la logique « organisation du chantier» puisse être capitalisée, connue et discutée par les garants de la logique « organisation de la continuité du fonctionnement de l'établissement » et vice versa. L'ergonome peut contribuer à influencer ces confrontations sociocognitives par l'action sur la conduite de projet. Sous réserve d'avoir les marges temporelles requises, il peut également assister à certaines réunions de chantier « stratégiques » pour informer les membres du comité de pilotage d'ajustements de «dernière minute » ayant des conséquences importantes sur le fonctionnement transitoire de l'établissement. Ces situations impliquent bien souvent, comme cela a été le cas sur le projet de l'unité gériatrique, des arbitrages définitifs du comité de pilotage afin que le chantier puisse poursuivre sa progression. 


\section{3.- Conclusion}

L'articulation entre permanence de fonctionnement d'un établissement et réalisation des travaux au cours d'une extension/restructuration architecturale sur un site occupé est un projet complexe. La cohabitation et la coordination de ces deux fonctionnements aux exigences propres constituent un déterminant important des formes possibles d'utilisation transitoire du bâtiment en travaux. Le coût humain associé invite à mieux prendre en compte dans le processus de conception l'ensemble des états transitoires d'une situation à transformer vers une situation transformée. Dans ce contexte, une démarche pour l'ergonome assistant de la Maîtrise d'Ouvrage a été proposée. Elle recouvre deux aspects complémentaires. D'une part la volonté que parmi les déterminants de la définition du phasage et des organisations transitoires apparaissent les situations d'utilisation réelles du bâtiment et ses exigences. D'autre part la volonté, malgré les obstacles identifiés, de favoriser les conditions favorables à la construction «d'un monde commun » dans lequel puisse s'exprimer et se confronter une diversité de logiques (continuité de fonctionnement, faisabilité et optimisation des travaux, etc.) jusqu'à la réception finale de l'ouvrage.

Plusieurs missions sont susceptibles d'être remplies par l'ergonome dans un contexte de travaux sur un site en fonctionnement:

- une mission d'assistance à la structuration de la conduite de projet du phasage et des organisations transitoires;

- une mission d'assistance à la validation du phasage ;

- une mission d'assistance à la définition et à l'ajustement des organisations transitoires.

L'enrichissement de ces missions et leur mise en œuvre à partir de nouvelles expériences de terrain permettraient d'approfondir cette première ébauche. Les ergonomes sont généralement appelés pour transformer les situations existantes ou participer à des projets de conception, mais ils sont beaucoup moins sollicités pour intervenir sur les situations de phasage et d'organisations transitoires. Ces situations intermédiaires sont vraisemblablement peu considérées du fait de leur caractère éphémère, pour autant, elles sont lourdes de conséquences sur la santé et l'efficacité des travailleurs.

\section{RÉFÉRENCES}

Argyris, C. (1983). Reasoning, Learning, and Action. San Francisco: Jossey-Bass Publishers.

Argyris, C., \& Schön, D. A. (1974). Theory in practice. Increasing Professional Effectivemeness. San Francisco: Jossey-Bass Publishers.

Argyris, C., \& Schön, D. A. (1978). Organization learning, a theory of action perspective. Reading MA: Addison-Wesley.

Argyris, C., \& Schön, D. A. (1996). Organization learning II. Reading MA: Addison-Wesley.

Armand, J., Raffestin, Y., \& Couffignal, D. (2001). Conduire son chantier. Paris: Le moniteur.

Béguin, P. (2007a). Innovation et cadre sociocognitif des interactions concepteurs-opérateurs : une approche développementale. Le travail humain, 70(4), 369-390.

Béguin, P. (2007b).Prendre en compte l'activité de travail pour concevoir. @ ctivités, 4(2), 107-114. http:// www.activites.org/v4n2/beguin-FR.pdf

Béguin, P., \& Cerf, M. (2004). Formes et enjeux de l'analyse de l'activité pour la conception de systèmes de travail. @ctivités, 1(1), 54-71. http://www.activites.org/v1n1/v1n1.pdf

Béguin, P., \& Pastré, P. (2002). Working, learning and designing through simulation. In S. P. Bagnara, A. Rizzo, \& P. Wright, Proceedings of the 11th European Conference on Cognitive Ergonomics : cognition, culture and design (pp. 5-13). 
Daniellou, F. (1992). Le statut de la pratique et des connaissances dans l'intervention ergonomique de conception. Bordeaux: Editions du Laboratoire d'Ergonomie des Systèmes Complexes, Université Victor Segalen Bordeaux 2.

Daniellou, F. (2004). L'ergonomie dans la conduite de projets de conception de systèmes de travail. In P. Falzon (Ed.), Ergonomie (pp. 359-373). Paris: PUF.

Darses, F., \& Reuzeau, F. (2004). Participation des utilisateurs à la conception des systèmes et dispositifs de travail. In P. Falzon (Ed.), Ergonomie (pp. 405-420). Paris: PUF.

Estingoy, P., \& Rabatel, M. (1994). Maîtrise d'Ouvrage Publique - Montage et suivi d'une opération de construction. Paris: Editions du Moniteur.

Garrigou, A., Daniellou, F., Carballeda, G., \& Ruaud, S. (1995). Activity analysis in participatory design and analysis of partipatory design activity. International Journal of Industrial Ergonomics, 15, 311-327.

Hatchuel, A. (1997). Coordination and control. In A. Sorge, \& M. Warner (Eds.), The Handbook of Organizational Behavior, International Encyclopedia of Business and Management (pp. 762-770). Lonodon: Thomson Business Press.

Jeantet, A., Tiger, H., \& Vinck, D. (1996). La coordination par les objets dans les équipes intégrées de conception de produit. In G. de Terssac, \& E. Friedberg (Eds.), Coopération et conception (pp. 87-100). Toulouse: Octarès.

Ledoux, E. (2000). Projets architecturaux dans le secteur sanitaire et social. Du bâtiment au projet : la contribution des ergonomes à l'instruction des choix. Bordeaux: Université Victor-Segalen, coll. "Thèses et Mémoires".

Maline, J. (1994). Simuler le travail, une aide à la conduite de projet. Paris: ANACT.

Martin, C. (1998). La conception architecturale entre volonté politique et faisabilité technique : le positionnement de l'intervention ergonomique. Thèse de doctorat d'ergonomie, Bordeaux : Université Victor Segalen Bordeaux 2.

Martin, C. (2004). L'ergonome dans les projets architecturaux. In P. Falzon (Ed.), Ergonomie (pp. 421-435). Paris: PUF.

Martin, C., Escouteloup, J., \& Daniellou, F. (1996). L'ergonome et la programmation architecturale. Performances Humaines et Techniques. Dossier architecture et ergonomie (9).

Pastré, P. (1999). Apprendre des situations. Editorial. Education Permanente, 139, 7-11.

Petit, J. (2005). Organiser la continuité du service : Intervention sur l'organisation d'une Mutuelle de santé. Thèse de doctorat en ergonomie, Université Victor Segalen Bordeaux 2, Editions du LESC.

Petit, J., \& Escouteloup, J. (2009). Intervention sur le phasage et le déroulement du chantier d'une maternité. Bordeaux: Rapport d'intervention. Département d'Ergonomie, ENSC, IPB.

Petit, J., Querelle, L., \& Daniellou, F. (2007). Quelles données pour la recherche sur la pratique de l'ergonome ? Le Travail Humain, 70(4), 391-411.

Saint-Arnaud, Y. (1992). Connaître par l'action. Montréal: PUF.

Senge, P. (2000). La cinquième discipline : le guide de terrain. Paris: Editions Générales First.

Six, F. (2004). La construction : le chantier au cœur du processus de conception-réalisation. In P. Falzon (Ed.), Ergonomie (pp. 633-646). Paris: PUF.

Six, F., \& Fourot, C. (2000). Le conducteur de travaux entre prescription du client, prescription de l'entreprise et conception - réalisation du chantier. 35e Congrès de la SELF "Communication et travail", (pp. 206-217). Toulouse.

Sousa Castro, I. (2010). A capitalização da experiência do uso do ambiente construido. Contribuições da Avaliação Pos-Ocupação e da Analise Ergonômica do trabalho. Estudo de caso realizado em um hospital-dia VIH. Thèse de doctorat. Université de Rio de Janeiro. 
Vicente, K. J. (1999). Cognitive work analysis : toward safe productive and healthy computer-based works. Lawrence Erlbaum Associate Publishers.

Vinck, D. (2009). De l'objet intermédiaire à l'objet-frontière. Vers la prise en compte du travail d'équipement. Revue d'anthropologie des connaissances, 3(1), 51-72.

RÉSUMÉ

Restructurations et extensions architecturales sur site occupé font partie des grandes classes de situation de conception. Elles posent le problème d'articulation entre la réalisation des travaux et la continuité de fonctionnement de l'établissement. Bien souvent, le phasage des travaux est défini par les concepteurs à partir des contraintes techniques et réglementaires. Dans de nombreux cas, les organisations transitoires nécessaires pour assurer un bon fonctionnement pendant le chantier ne sont pas anticipées. L'ergonomie s'est beaucoup intéressée à la compréhension de l'évolution d'une situation A vers une situation B transformée dans un contexte architectural. Elle s'est moins préoccupée de la compréhension des états transitoires entre ces deux pôles et ses conséquences sur le plan de la santé des utilisateurs et la performance de l'établissement. A partir de diverses interventions réalisées dans ces circonstances, cet article propose une démarche possible de l'ergonome pour aider à concilier au mieux permanence du fonctionnement et travaux: 1) favoriser la rencontre des mondes professionnels; 2) contribuer à instruire les grandes orientations de phasage; 3) accompagner la définition des organisations transitoires par le monde des utilisateurs; 4) favoriser l'ajustement des organisations transitoires pendant le chantier.

\section{MotS CLÉS}

Pratique réflexive, intervention ergonomique, conception architecturale, phasage des travaux, organisations transitoires, mondes professionnels.

\section{RÉFÉRENCEMENT}

Beaujouan, J., Escouteloup, J, \& Daniellou, F. (2011). Phasage des travaux et organisations transitoires: Quels rôles pour l'ergonome? Activités, 8(1), pp. 26-43, http://www.activites.org/v8n1/v8n1.pdf

Article soumis le 14 octobre 2010, accepté pour publication le 18 janvier 2011 